\title{
Analytical Chemistry by Electrophoresis
}

\author{
Yukihiro Окамото \\ Division of Chemical Engineering, Graduated School of Engineering Science, Osaka University, \\ 1-3 Machikaneyama-cho, Toyonaka, Osaka 560-8531, Japan
}

Electrophoresis enables high-performance analysis concerning from small-to-large molecules, dissolved molecules to insoluble particles, and charged to neutral molecules in a low-cost and easy manner.

Slab gel electrophoresis is one of the most established methods in electrophoresis. Agarose and poly(acrylamide) gels electrophoresis have been used for nucleic acids ${ }^{1}$ and protein separation $^{2}$ due to no electroosmotic flow and non-specific interactions between gels. Indeed, Hwang and Choi reported on the separation of phosphoproteins by sodium dodecyl sulfatepoly(acrylamide) gel electrophoresis and their specific detection with a fluorescence sensor, 1-(2-hydroxy-1-naphthylazo)-2naphthol-4-sulfonic acid sodium salt (Calcon). ${ }^{3}$ In addition, $\mathrm{Gu}$ et al. reported on analysis by the Western blot technique for the silk identification of different species. ${ }^{4}$

In the case of samples with similar electrophoretic mobility, the addition of affinity molecules is effective to alter the electrophoretic mobility and to separate the target molecules, specifically. Sato et al. reported on the boronate affinity electrophoresis for the separation of PIWI-interacting RNA (piRNA) from Mouse Testis Total RNA. ${ }^{5}$ These papers represented that slab gel electrophoresis has been employed routinely in academic research and as practical applications

Capillary electrophoresis (CE) ${ }^{6}$ and microchip electrophoresis $(\mathrm{MCE})^{7}$ enable automatic analysis, and MCE especially enables micro-total analysis. $\mathrm{CE}$ and MCE, which uses a micrometersized capillary and a microchannel, do not require large volume samples, and attain superior separation because of plug flow, suppression of convection, as well as heat dissipation. These characters are favorable in high-throughput analysis and

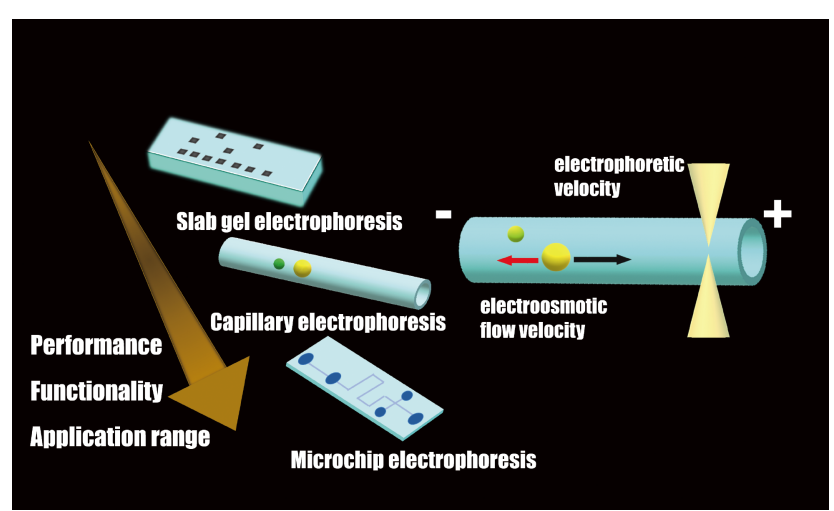

Various electrophoresis style and electrophoretic behavior in aqueous solution.

E-mail: okamoto@cheng.es.osaka-u.ac.jp diagnosis. ${ }^{8-11}$ Furthermore, by adding affinity molecules to buffer solution or by constructing a stationary phase in the capillary or microchannel, neutral molecules and enantiomers, which have the same electrophoretic mobility, can be separated due to different interactions between the (pseudo) stationary phase. ${ }^{12,13}$ Liu et al. immobilized Janus structured $\mathrm{Au}-\mathrm{Fe}_{3} \mathrm{O}_{4}$ nanoparticles on the capillary and separated dihydroxy benzene isomers. ${ }^{14}$

In addition to separation tools, $\mathrm{CE}$ and MCE are useful for the analysis of molecular interactions because $\mathrm{CE}$ and MCE do not require the immobilization of affinity ligands, and enable one to analyze the molecular interaction in a natural state, that is, an aqueous soluble state. Focusing on the advantages of CE and MCE, Miyabe reported on a moment analysis of mass transfer kinetics in a micellar. ${ }^{15}$ Takayanagi et al. reported on the electrophoretic behavior of graphene, demonstrating that the dispersion of graphene in an aqueous solution can be monitored and that surfactant on the graphene surface is replaced competitively by water-soluble nonionic polymers. ${ }^{16}$

$\mathrm{CE}$ and MCE can be connected with various detection methods ${ }^{17-20}$ and analysis methods, such as mass spectrometry. This merit affords fruitful information about molecular species in a sample. On the other hand, the disadvantage of CE and MCE is low sensitivity. To overcome this problem, highly sensitive detection methods, such as laser-induced fluorescence, ${ }^{20}$ preconcentration with a solid-phase extractant, ${ }^{21}$ and on-line concentration ${ }^{22-24}$ have been adopted.

In this highlight, recent analysis by electrophoresis is briefly reviewed. Slab gel electrophoresis is now routinely employed in bioanalysis. CE and MCE have expanded the application of electrophoresis from large (nucleic acids, proteins) to small (organic, inorganic molecules) including neutral molecules and enantiomers, and from water-soluble to insoluble (nanoparticles, liposomes, cells etc.). In addition, various analysis methods based on electrophoresis have been reported, such as dielectrophoresis ${ }^{25}$ and electrophoresis in a lipid membrane. ${ }^{26}$ Therefore, the importance of electrophoretic analysis will continue to increase steadily.

Keywords Slab gel electrophoresis, capillary electrophoresis, microchip electrophoresis

\section{References}

1. C. Liu, Z. Hu, X. Wang, Y. Geng, C. Ma, Z. Wang, R. Li, and C. Shi, Anal. Sci., 2019, 35, 449.

2. S. B. Kim, R. Nishihara, R. Fujii, R. Paulmurugan, D. Citterio, and K. Suzuki, Anal. Sci., 2019, 35, 71 .

3. S. Y. Hwang and J. K. Choi, Anal. Sci., 2018, 34, 1427.

4. J. Gu, C. Xu, M. Li, B. Chen, Y. Shang, H. Zheng, Y. Zhou, 
Z. Hu, Z. Peng, and B. Wang, Anal. Sci., 2019, 35, 175.

5. Y. Sato, D. Iwasawa, K. P. Hui, R. Nakagomi, and S. Nishizawa, Anal. Sci., 2018, 34, 627.

6. R. L. C. Voeten, I. K. Ventouri, R. Haselberg, and G. W. Somsen, Anal. Chem., 2018, 90, 1464.

7. M. Yang, Z. Huang, J. Chang, and H. You, Anal. Sci., 2018, 34, 1285.

8. Y. Esaka, K. Hisato, T. Yamamoto, H. Murakami, and B. Uno, Anal. Sci., 2018, 34, 901.

9. Y. Yagi, A. Okazaki, M. Endo, K. Yanagisawa, J. Fukuda, K. Nishimura, and K. Yamazaki, Anal. Sci., 2019, 35, 1333.

10. K. Wakui, A. Abe, T. Yoshitomi, H. Furusho, and K. Yoshimoto, Anal. Sci., 2019, 35, 585.

11. D. Goto, K. Ouchi, M. Shibukawa, and S. Saito, Anal. Sci., 2015, 31, 1143.

12. S. Terabe, K. Otsuka, K. Ichikawa, A. Tsuchiya, and T. Ando, Anal. Chem., 1984, 56, 111.

13. Y. Okamoto, Y. Ikawa, F. Kitagawa, and K. Otsuka, J. Chromatogr., A, 2007, 1143, 264.

14. Y. Liu, J. Li, Y. Wang, and C. Yan, Anal. Sci., 2020, 36, 413.

15. K. Miyabe and N. Suzuki, Anal. Sci., 2018, 34, 215.
16. T. Takayanagi, Y. Becchaku, Y. Tomiyama, M. Kurashina, and H. Mizuguchi, Anal. Sci., 2019, 35, 307.

17. Z. Huang, M. Yang, H. You, and Y. Xie, Anal. Sci., 2018, 34, 801 .

18. S. Yamamoto, H. Fujiwara, K. Maruyama, Y. Tanaka, M. Kinoshita, and S. Suzuki, Anal. Sci., 2019, 35, 295.

19. Y. Tan, Q. Song, W. Liu, M. Li, J. Xiao, and C. Chen, Anal. Sci., 2019, 35, 1103.

20. J. Mbuna and T. Kaneta, Anal. Sci., 2015, 31, 1121.

21. Y. Okamoto, F. Kitagawa, and K. Otsuka, Anal. Chem., 2007, 79, 3041.

22. F. Kitagawa, S. Wakagi, Y. Takegawa, and I. Nukatsuka, Anal. Sci., 2019, 35, 889.

23. A. V. Ivanov, K. A. Nikiforova, P. O. Bulgakova, E. D. Virus, and A. A. Kubatiev, Anal. Sci., 2018, 34, 505.

24. H. Umeda, S. Kitagawa, and H. Ohtani, Anal. Sci., 2015, $31,1151$.

25. T. Yasukawa, A. Morishima, M. Suzuki, J. Yoshioka, K. Yoshimoto, and F. Mizutani, Anal. Sci., 2019, 35, 895.

26. Y. Okamoto, Y. Tsujimoto, and H. Umakoshi, Electrophoresis, 2016, 37, 762. 